\title{
Grape seed proanthocyanidins protect against streptozotocin-induced diabetic nephropathy by attenuating endoplasmic reticulum stress-induced apoptosis
}

\author{
ZHAOLI GAO $^{1 *}$, GUANGYI LIU ${ }^{2,3 *}$, ZHAO HU $^{2}$, WEIWEI SHI ${ }^{4}$, BINBIN CHEN $^{5}$, PEIMEI ZOU $^{6}$ and XIANHUA ${ }^{2}$ \\ ${ }^{1}$ Department of Nephrology, Shandong University Qi Lu Hospital Qing-Dao, Qingdao, Shandong 266000; \\ ${ }^{2}$ Department of Nephrology, Shandong University Qi Lu Hospital; ${ }^{3}$ Ministry of Education Key Laboratory of \\ Experimental Teratology and Institute of Molecular Medicine and Genetics, Shandong University School of Medicine; \\ ${ }^{4}$ Department of Ultrasound, Jinan Central Hospital of Shandong University, Jinan, Shandong 250012; \\ ${ }^{5}$ Department of Nephrology, Liaocheng People's Hospital, Liaocheng, Shandong 252000; \\ ${ }^{6}$ Department of Nephrology, Peking Union Medical College Hospital, Beijing 100000, P.R. China
}

Received November 21, 2017; Accepted May 11, 2018

DOI: $10.3892 / \mathrm{mmr} .2018 .9140$

\begin{abstract}
Diabetic nephropathy (DN) is by far the most common cause of end-stage renal disease (ESRD) in industrial countries, accounting for $\sim 45 \%$ of all new ESRD cases in the United States. Grape seed proanthocyanidin extracts (GSPE) are powerful antioxidants, with an antioxidant ability 50 -fold greater than that of vitamin $\mathrm{E}$ and 20 -fold greater than that of vitamin $\mathrm{C}$. The present study investigated whether GSPE can protect against streptozotocin (STZ)-induced DN and aimed to elucidate a possible mechanism. Male Sprague Dawley rats were randomly divided into three groups: Control group (N), diabetes mellitus group (DM) injected with $40 \mathrm{mg} / \mathrm{kg} \mathrm{STZ \text {, }}$ and the GSPE treatment group (intragastric administration of $250 \mathrm{mg} / \mathrm{kg} /$ day GSPE for 16 weeks after diabetes was induced in the rats). Blood and kidney samples were collected after treatment. The renal pathological changes were determined with periodic acid-Schiff (PAS) staining, while the protein expression levels of glucose-regulated protein 78 (GRP78), phosphorylated-extracellular signal-regulated kinase (p-ERK)
\end{abstract}

Correspondence to: Professor Xianhua Li, Department of Nephrology, Shandong University Qi Lu Hospital, 107 Wenhua Xi Road, Jinan, Shandong 250012, P.R. China

E-mail: lixianhua7075@sina.com

*Contributed equally

Abbreviations: GSPE, grape seed proanthocyanidin extracts; STZ, Streptozotocin; DN, diabetic nephropathy; ER, endoplasmic reticulum; GRP78, glucose-regulated protein 78; p-ERK, phosphorylated-extracellular signal-regulated kinase; BUN, blood urea nitrogen; Scr, serum creatinine; RI, renal index; ESRD, end stage renal disease

Key words: grape seed proanthocyanidins, streptozotocin, diabetic nephropathy, endoplasmic reticulum stress, apoptosis and Caspase-12 were determined by western blotting and immunohistochemical staining. Apoptosis was determined with a terminal deoxynucleotidyl transferase dUTP nick-end labeling (TUNEL) assay. Compared with the DM group, the GSPE group had no significant changes in the blood urea nitrogen (BUN) level and serum creatinine (Scr) level, but showed a significant decline in the renal index (RI) level and 24-h urinary albumin level $(\mathrm{P}<0.05)$. The histopathology results indicated very little pathological damage in the GSPE group. Compared with the DM group, the GSPE group had a significantly reduced number of TUNEL-positive cells $(\mathrm{P}<0.05)$, and the GSPE group had an obvious reduction in the protein expression of GRP78, p-ERK, and Caspase-12 $(\mathrm{P}<0.05)$. In this study, the results indicated that GSPE can protect renal function and attenuate endoplasmic reticulum stress-induced apoptosis via the Caspase-12 pathway in STZ-induced DN.

\section{Introduction}

Diabetic nephropathy (DN) is by far the most common cause of end-stage renal disease (ESRD) in industrial countries, making up approximately $45 \%$ of all new ESRD cases in the United States (1). Its pathogenesis is a chronic and complex process. At the later stages of DN, the gradual decline of renal function, tubular atrophy and interstitial fibrosis occurs (2). Several studies have shown that apoptosis could be considered a vital component in the processes of DN (3-6). However, several pathways can induce apoptosis, including the intrinsic pathway, the extrinsic pathway and the endoplasmic reticulum stress (ERS) pathway (7).

The ER is the site of lipid biosynthesis, protein folding and protein maturation in eukaryotic cells. The ER is extremely sensitive to the factors that affect intracellular energy levels, the oxidation state, and the calcium concentration. When the cells receive a shock (such as from hypoxia, and toxic drugs), the ER environment is destroyed, inducing calcium metabolism disorder, ER function disorder, an increase in 
unfolded or misfolded proteins in the ER, and calcium imbalance. We designated this state as ERS. One major response of ERS is dissociation of glucose-regulated protein 78 (GRP78) with transmembrane receptor which leads to its activation to deal with the accumulated unfolded proteins (8). Phosphorylated-extracellular signal-regulated kinase (p-ERK) is transmembrane protein in the ER, which plays a signal transduction role. $\mathrm{p}-\mathrm{ERK}$ is known as an initial, crucial protector for survival during even mild stress (9). Slight and medium ERS can protect the cell from death, but severe ERS induces Caspase-12-dependent cell apoptosis (9). Since the ERS has both protective and deleterious features, a better understanding of the molecular pathways of the ERS could reveal novel therapeutic strategies in chronic renal diseases, including diabetic kidney disease.

Grape seed proanthocyanidin extracts (GSPE) are derived from grape seeds and have been shown to possess potent antioxidant, anti-inflammatory, radical-resistance, anti-tumor, and cardiovascular protecting properties (10-12). In this study, we treated a streptozotocin (STZ)-induced DN rat model with GSPE to investigate the effect of GSPE on STZ-induced DN. In order to ascertain whether a protective effect of GSPE on DN occurred through the inhibition of ERS-induced apoptosis, We investigated the protein expression levels of GRP78, p-ERK and Caspase-12 by Western blotting and immunohistochemical staining. We used TUNEL kit to detect apoptosis.

\section{Materials and methods}

Reagents. Grape seed proanthocyanidin (purity exceeds $96 \%$, Lot no. G050412) was purchased from Tianjin Jianfeng Natural Product R\&D Co., Ltd. (Tianjin, China). STZ was purchased from Sigma. TUNEL staining kit (in situ Cell Death Detection kit) was obtained from Roche Diagnostics, (Indianapolis, IN, USA). Primary antibodies used in this study include: Rabbit anti-Caspase-12, rabbit anti-GRP78 (Abcam Ltd., Hong Kong, China), rabbit anti-p-ERK, rabbit anti-ERK antibodies (Cell Signaling Technology, Inc., Danvers, MA, USA), mouse anti- $\beta$-actin (Santa Cruz Biotechnology, Inc., Dallas, TX, USA). Anti-mouse and anti-rabbit secondary antibodies were purchased from Jackson ImmunoResearch Laboratories, Inc. (West Grove, PA, USA).

Animals. Fifty-five adult male SD rats (8-12 weeks of age), weighing 120-160 g were provided by the Beijing Vital River Laboratory Animal Technology Co., Ltd (Beijing, China). The current study was approved by the Animal Ethics Committee of Shandong University (no. DWLL-2013-053; Jinan, China).

Forty-five randomly selected rats were given a high-fat diet $(45 \%$ fat, $20 \%$ protein and $35 \%$ carbohydrate, as a percentage of total kcal; product no. D12451, provided by the Beijing Vital River Laboratory Animal Technology Co., Ltd.) for one month, and then intraperitoneally injected with $40 \mathrm{mg} / \mathrm{kg} \mathrm{STZ}$ dissolved in $\mathrm{pH} 4.5$ citrate buffer, while the ten remaining rats were given the same dosage of citrate buffer at $\mathrm{pH} 4.5$ and given a normal diet ( $\mathrm{N}$ group). Hyperglycemia was confirmed by measuring the venous circulating plasma concentration of glucose. Seven days post-STZ injection, blood samples were obtained from the rat tail vein after $12 \mathrm{~h}$ of fasting, and the glucose concentration was determined with an automatic analyzer (a Comfore automatic analyzer purchased from Shanghai). Forty rats showed the diabetic rat standard of a fasting blood glucose level higher than $300 \mathrm{mg} / \mathrm{dl}$, while the value in the control group of rats injected with citrate buffer ranged from 90 to $130 \mathrm{mg} / \mathrm{dl}$. Then, twenty randomly selected rats were continued on the high-fat diet (DM group) until 16 weeks, and the other twenty rats received intragastric administration of $250 \mathrm{mg} / \mathrm{kg} /$ day GSPE as well as the high-fat diet (GSPE group) until 16 weeks. At the end of the experiment, 16 rats survived in the DM group and 15 in the GSPE group. Three rats in the GSPE group died of asphyxiation due to gavage errors. The animals were placed in individual metabolic cages for $24 \mathrm{~h}$ to collect urine samples before the experimental rats were sacrificed. No difference of total water intake among groups was observed. Total urinary protein $(\mathrm{g} / \mathrm{l})$ was measured by the sulfosalicylic acid method (722N; China) (13). At the end of the experiments, the animals were fasted overnight for $18 \mathrm{~h}$ and then anesthetized with intraperitoneal injection of sodium pentobarbitone $(60 \mathrm{mg} / \mathrm{kg}$; Sigma-Aldrich; Merck KGaA, Darmstadt, Germany) and sacrificed. All animals were weighed before sacrifice. Blood was collected from the heart before sacrifice, and the serum was separated by centrifugation $\left(912 \mathrm{x} \mathrm{g}\right.$ at $4^{\circ} \mathrm{C}$ for $\left.20 \mathrm{~min}\right)$ and stored at $-80^{\circ} \mathrm{C}$ until it was assayed. Each kidney was cut in half by coronal plane after excision and weighed. Two pieces of kidney were stored at $-80^{\circ} \mathrm{C}$ for a Western blotting analysis. The other pieces were fixed in $4 \%$ buffered paraformaldehyde at $4^{\circ} \mathrm{C}$ and embedded in paraffin for immunohistochemical histopathologic observation, and a terminal deoxynucleotidyl transferase dUTP nick-end labeling (TUNEL) assay.

Assessments of renal function. Blood urea nitrogen (BUN) and serum creatinine (Scr) were analyzed on a Cobas 8000 (Roche Diagnostics) in Qilu Hospital, Shandong University. The renal index (RI) was calculated as follows: both kindey weights (g)/animal's weight $(\mathrm{g})$ x100.

Histopathologic and Immunohistochemical staining. The renal pathological changes were determined by periodic acid-Schiff (PAS) staining. The kidneys were sliced and fixed in $4 \%$ paraformaldehyde for no more than $24 \mathrm{~h}$ and then taken to $0.5 \%$ paraformaldehyde for long-term preservation. They were embedded in paraffin and cut into $4-\mu \mathrm{m}$-thick sections for staining with PAS or immunohistochemical staining.

The PAS-positive area present in the mesangial region excluding cellular elements indicated mesangial matrix expansion. The percentage of the PAS-positive area in the glomerulus was analyzed by using Leica QWin version 3 image analysis software (Leica Microsystems GmbH, Wetzlar, Germany).

For immunohistochemical analysis, the tissue slices were microwaved for 10 to $15 \mathrm{~min}$ in $0.01 \%$ sodium citrate buffer (pH 6.0) for antigen retrieval. The tissue slices were cooled at room temperature or in ice water and then washed with PBS three times. The tissue slices were immersed in $0.1 \%$ Triton X-100 for $15 \mathrm{~min}$. To block endogenous peroxidase, all tissue slices were incubated with $3 \%$ hydrogen peroxide for $10 \mathrm{~min}$ in the dark. The tissue slices were incubated with $10 \%$ goat serum for $60 \mathrm{~min}$ at $37^{\circ} \mathrm{C}$, then with primary antibody at $4^{\circ} \mathrm{C}$ overnight (anti-GRP78 1:200, anti-p-ERK 1:50, anti-Caspase-12 1:100). The negative control sections 
Table I. Blood glucose of animals.

\begin{tabular}{lcccc}
\hline & \multicolumn{4}{c}{ Blood glucose $(\mathrm{mmol} / \mathrm{l})$} \\
\cline { 2 - 5 } Group & 4 weeks & 8 weeks & 12 weeks & 16 weeks \\
\hline $\mathrm{N}$ & $7.05 \pm 0.73$ & $7.24 \pm 0.46$ & $7.33 \pm 0.47$ & $7.04 \pm 1.06$ \\
$\mathrm{D}$ & $7.74 \pm 1.09$ & $25.28 \pm 4.50^{\mathrm{a}}$ & $24.23 \pm 4.21^{\mathrm{a}}$ & $26.49 \pm 3.14^{\mathrm{a}}$ \\
$\mathrm{G}$ & $7.74 \pm 1.09$ & $26.15 \pm 4.38^{\mathrm{a}}$ & $24.14 \pm 5.03^{\mathrm{a}}$ & $24.89 \pm 3.05^{\mathrm{a}}$ \\
\hline
\end{tabular}

${ }^{a} \mathrm{P}<0.05$ compared with the $\mathrm{N}$ group. Data are presented as means \pm standard deviation. $\mathrm{N}$, the control group; $\mathrm{D}$, the diabetes mellitus group; $\mathrm{G}$, the grape seed proanthocyanidin extracts group.

Table II. Animal data and biochemical parameters.

\begin{tabular}{|c|c|c|c|c|c|}
\hline Group & $\mathrm{n}$ & RI & Pro $\left(\times 10^{-3} \mathrm{~g}\right)$ & BUN (mmol/l) & $\operatorname{Scr}($ umol/l) \\
\hline $\mathrm{N}$ & 10 & $0.64 \pm 0.036$ & $5.48 \pm 1.36$ & $6.28 \pm 0.43$ & $50.2 \pm 7.79$ \\
\hline $\mathrm{D}$ & 16 & $1.07 \pm 0.074^{\mathrm{a}}$ & $44.82 \pm 5.39^{\mathrm{a}}$ & $5.84 \pm 0.81$ & $49.63 \pm 7.21$ \\
\hline G & 15 & $0.98 \pm 0.085^{\mathrm{a}, \mathrm{b}}$ & $35.12 \pm 6.89^{\mathrm{a}, \mathrm{b}}$ & $5.79 \pm 0.70$ & $48.00 \pm 5.29$ \\
\hline
\end{tabular}

${ }^{a} \mathrm{P}<0.05$ compared with the $\mathrm{N}$ group. ${ }^{\mathrm{b}} \mathrm{P}<0.05$ compared with the $\mathrm{D}$ group. Data were presented as means \pm standard deviation. $\mathrm{n}$, the number of the survived animal in each group; RI, renal index; BUN, blood urea nitrogen level; Scr, serum creatinine level; Pro, the lever of $24 \mathrm{~h}$ urinary album in; N, the control group; D, the the diabetes mellitus group; $\mathrm{G}$, the grape seed proanthocyanidin extracts group.

were incubated with PBS instead of the primary antibody. All sections were incubated with secondary antibodies for $60 \mathrm{~min}$ at $37^{\circ} \mathrm{C}$ and then stained with DAB and hematoxylin. The stained slides were analyzed by light microscopy. Brown areas were designated as positive. A semi-quantitative analysis was performed on the colored sections using an Image-Pro Plus 5.0.

TUNEL staining. TUNEL staining was performed according to the manufacturer's instructions. The sections were incubated with proteinase $\mathrm{K}$ at $37^{\circ} \mathrm{C}$ for $15 \mathrm{~min}$, then washed in PBS for $5 \mathrm{~min}$; the enzyme solution and label solution were then mixed (1:9) at $37^{\circ} \mathrm{C}$ for $60 \mathrm{~min}$ in the dark. The sections immersed in label solution served as negative controls. The sections were then stained with DAPI for $10 \mathrm{~min}$. TUNEL-positive nuclei were expressed as a percentage of the total nuclei per field. Ten fields per section and two sections per kidney were assayed in each experiment.

Proteins sample preparation. The tissue samples were homogenized in TRIzol (50-100 mg tissue per $1 \mathrm{ml}$ TRIzol). After chloroform was added (0.2 $\mathrm{ml}$ chloroform per $1 \mathrm{ml}$ TRIzol), the homogenates were centrifuged at $12,000 \mathrm{x} g$ for $15 \mathrm{~min}$ at $4^{\circ} \mathrm{C}$. The supernatants were discarded, isopropanol was added (1.5 $\mathrm{ml}$ isopropanol per $1 \mathrm{ml}$ TRIzol) to the lower phase, and then the samples were centrifuged at $12,000 \mathrm{x}$ g for $15 \mathrm{~min}$ at $4^{\circ} \mathrm{C}$. The pellets were washed with $0.3 \mathrm{M}$ guanidine hydrochloride ( $2 \mathrm{ml}$ guanidine hydrochloride per $1 \mathrm{ml}$ TRIzol) three times, then dissolved in $1 \%$ SDS (100 $\mu \mathrm{l}$ SDS per $1 \mathrm{ml}$ TRIzol) at $50^{\circ} \mathrm{C}$ for $30 \mathrm{~min}$. The concentration of protein was determined using the Pierce BCA assay kit.
Western blotting. Protein $(50 \mu \mathrm{g})$ was subjected to $10 \%$ or $12 \%$ SDS-polyacrylamide gel electrophoresis and transferred to cellulose acetate membranes. The membranes were blocked at room temperature for $1 \mathrm{~h}$ or more with $5 \%$ skim milk, then incubated with the primary antibodies (anti-GRP78 1:250, anti-p-ERK 1:1,000, anti-ERK 1:1,000, anti-caspase 12 1:500, anti- $\beta$-actin $1: 2,500)$ at $4{ }^{\circ} \mathrm{C}$ overnight. After incubation with the secondary antibodies at room temperature for $1 \mathrm{~h}$, the membranes were soaked with enhanced chemiluminescence (ECL) reagent and exposed to X-ray film. Quantification of the luminosity of each identified protein band was performed using Adobe Photoshop software (Adobe Photoshop 7.0; Adobe, San Jose, CA, 2002). The Western blotting analysis used a ratio of GRP78, p-ERK, and Caspase-12 in the $\mathrm{N}$ group to the other groups.

Statistical analysis. Data were displayed as means \pm standard deviation. Differences between groups were analyzed using the post hoc test used for multiple comparisions following one-way ANOVA. $\mathrm{P}<0.05$ was considered to indicate a statistically significant difference.

\section{Results}

GSPE protects against STZ-induced DN. As shown in Table I, the blood glucose in the DM group and the GSPE group was higher during the entire experiment, and it was statistically significant compared with the control group. As shown in Table II, 10 rats survived in the $\mathrm{N}$ group, 16 rats survived in the DM group, and 15 rats survived in the GSPE group at the end of the study. The BUN level and Scr had 

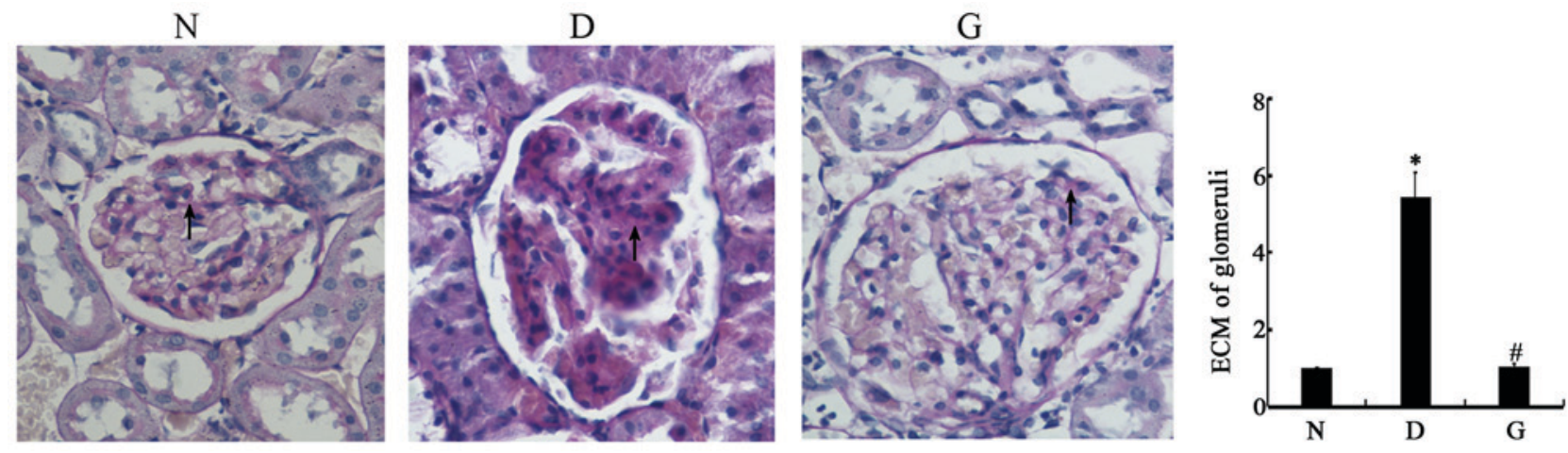

Figure 1. GSPE protects Streptozotocin-induced diabetic nephropathy. Animals were divided into three group: The control group (N), the DM group (D), the GSPE group (G), Sections were stained with PAS and were examined by microscopy. Original magnification, x400. The glomerular hypertrophy, thickness of the glomerular basement membrane in the D group can be found. In $\mathrm{G}$ group, the change of glomerular was significant reduced. The histogram means the relative $\mathrm{ECM}$ accumulation of each group to the $\mathrm{N}$ group, $\mathrm{N}$ group was set to 1 . The $\mathrm{ECM}$ in the $\mathrm{D}$ group is $5.46 \pm 0.61$, and in the $\mathrm{G}$ group is $1.04 \pm 0.09$. Higher ECM accumulation was observed in the kidneys of $\mathrm{D}$ group rats compared with that of the $\mathrm{N}$ group, Lower ECM accumulation was observed in the kidneys of the $\mathrm{G}$ group rats compared with the $\mathrm{D}$ group. " $\mathrm{P}<0.05$ compared with the $\mathrm{N}$ group; ${ }^{*} \mathrm{P}<0.05$ compared with the $\mathrm{D}$ group. DM, diabetes mellitus; GSPE, grape seed proanthocyanidin extracts; PAS, periodic acid-Schiff; ECM, extracellular matrix.

no significant changes among the three groups, but the 24-h urinary albumin level and the RI increased in the DM group compared with the $\mathrm{N}$ group. Compared with the DM group, the level of 24-h urine albumin and RI significantly decreased in the GSPE group.

The histopathology results are shown in Fig. 1. PAS staining indicated the presence of glomerular hypertrophy and thickening of the glomerular basement membrane in the DM group, which showed that the diabetic rats suffered from diabetic nephrology. In the GSPE group, the glomerular changes were significantly reduced, which indicated that the GSPE had a protective effect against diabetic nephrology. Quantitative analysis of the percentage of the PAS-positive area in the glomeruli is summarized in the histogram. The extracellular matrix (ECM) accumulation was significantly higher in the glomeruli of the $\mathrm{D}$ group than that of the $\mathrm{N}$ group $(P<0.05)$, and the $G$ group was significantly shorter than that of the $\mathrm{D}$ group $(\mathrm{P}<0.05)$.

GSPE inhibits apoptosis in the DM group rats. To assess whether GSPE inhibited cell apoptosis in DN, the tissue sections were labeled with an in situ TUNEL assay. As shown in Fig. 2, apoptotic cells were visible with a red color. The DM group had high expression of TUNEL-positive cells compared with the $\mathrm{N}$ group $(\mathrm{P}<0.05)$. In the GSPE group the TUNEL-positive cells were significantly reduced compared with the DM group $(\mathrm{P}<0.05)$. In the $\mathrm{N}$ group and the GSPE group, little apoptosis was evident.

GSPE inhibits the expression of GRP78, p-ERK and caspase-12. Apoptosis occurred in the cells with DN. To investigate whether GSPE protects against DN by attenuating ERS-induced apoptosis, we determined the expression of p-ERK, GRP78, and Caspase-12, important participants in ERS-induced apoptosis, by Western blotting and immunohistochemical methods. The two approaches showed that GRP78, p-ERK and Caspase-12 were highly expressed in the DM group, while their levels were significantly reduced in the GSPE group $(\mathrm{P}<0.05$; Figs. 3 and 4$)$. These proteins showed limited expression in the $\mathrm{N}$ group.

\section{Discussion}

Diabetes mellitus (DM) is a multifactorial chronic metabolic disease characterized by hyperglycaemia (14). DM can be divided by the etiology type into type 1 diabetes (T1DM), type 2 diabetes (T2DM) and other special types of diabetes, and approximately $90-95 \%$ of diabetic patients have T2DM. T2DM is the most common form of diabetes, and it is caused by genetic and environmental factors. Patients with T2DM develop DN after 15 years (15). DN is one of the most serious complications of diabetes, and it is by far the most common cause of ESRD in industrial countries (16). It has been shown that several genetic and environmental factors probably contribute to its development, although the precise mechanisms are unknown (17). Accumulating evidence suggests that ERS-induced apoptosis may play an important role in the progression of DM and its complications (14).

In our study, we first established a model of type 2 diabetes. The establishment of a T2DM model can be induced with a high-fat diet for a period of time to induce insulin resistance, and then a small dose of intraperitoneal injection of STZ is given to destroy the islets, resulting in insulin secretion deficiency-induced hyperglycemia disease, in order to mimic the pathogenesis of human type 2 diabetes (18). The high-fat diet and low-dose STZ-induced rat model was initially developed by Reed et al (19) and modified by Srinivasan et al (20), and it closely mimics the natural history of human metabolic syndrome and T2DM. In this study, the DM group showed a significant increase in the level of 24-h urine albumin and RI compared with the $\mathrm{N}$ group. The histopathology results showed that the DM group had significant structural glomerular damage compared with the $\mathrm{N}$ group. The number of TUNEL-positive cells was significantly increased in the DM group compared with the $\mathrm{N}$ group. However, compared with the DM group, the GSPE group showed a significant decline in the level of 24-h urine albumin and RI. The histopathology results also showed that the GSPE group had very little glomerular damage. These observations are consistent with previous studies (12). It has been shown that GSPE is safe in animals with normal kidneys (12). Compared with the DM 

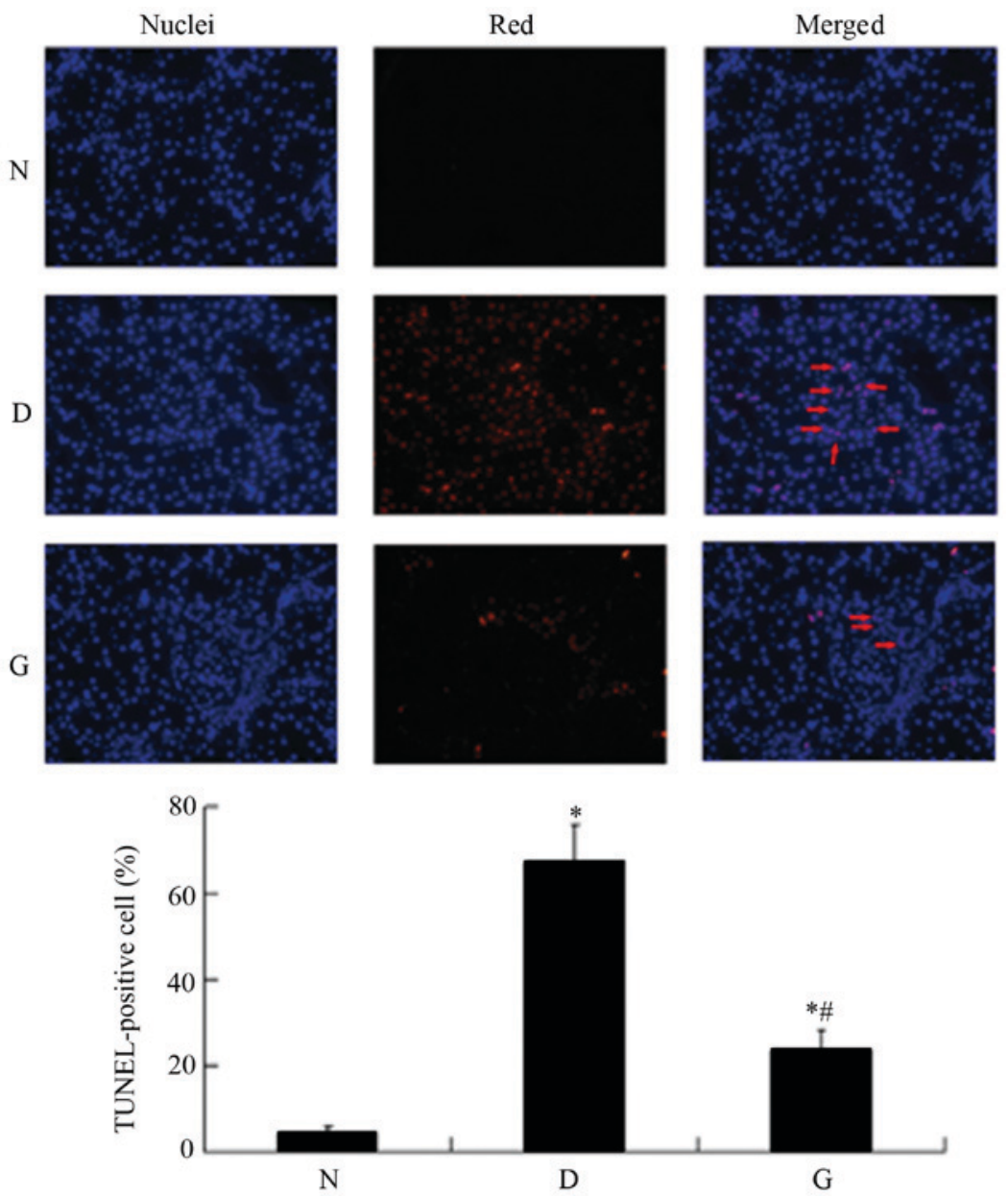

Figure 2. GSPE inhibits apoptosis in DM group rats. Arrows are representative of TUNEL-positive cells. Original magnification, $x 400$. Animals were divided into three group: The control group (N), the DM group (D), the GSPE group $(\mathrm{G})$. Data are presented as means $\pm \mathrm{SD}$. * $\mathrm{P}<0.05$ compared with the $\mathrm{N}$ group; ${ }^{\#} \mathrm{P}<0.05$ compared with the D group. DM, diabetes mellitus; GSPE, grape seed proanthocyanidin extracts; TUNEL, terminal deoxynucleotidyl transferase dUTP nick-end labeling.

group, the GSPE group had a significant decline in the number of TUNEL-positive cells, which showed that GSPE can inhibit apoptosis in DN, reduce the generation of proteinuria and delay the development of kidney damage. This phenomenon is associated with the antioxidant activities of GSPE, for which it has been shown that oxidative stress proteins can be down-regulated by GSPE in DN $(11,12)$. Recent studies have shown that GSPE has powerful antioxidant activity. Its antioxidative activity is much stronger than that of vitamin $\mathrm{C}$ and vitamin E (12). Additionally, GSPE has an anti-apoptosis function in diabetes (21). Some recent studies have found that apoptosis caused by the ERS pathway plays an important role $(4,9)$. Studies have even shown that apoptosis induced by ERS is involved in diabetic kidney disease (4). However, whether GSPE can protect against STZ-induced DN by attenuating ERS-induced apoptosis is unknown, so we investigated it in the present study.

A variety of factors, including ischemia, hypoxia, glucose starvation, heat shock and $\mathrm{Ca}^{2+}$ overload, can disturb ER function and result in ERS (9). Gentle ERS can protect cells from damage, but prolonged or severe stress leads to apoptosis (22-24). ERS is evident in various renal diseases, including primary glomerulonephritides, acute kidney injury, chronic kidney disease, renal fibrosis, glomerulopathies associated with genetic mutations and DN. The induction of ERS may be cytoprotective, or it may be cytotoxic by activating apoptosis $(25,26)$. In mammalian cells, there are three major arms of the ERS: PERK, inositol-requiring protein-1 (IRE1), and activating transcription factor-6 (ATF6) pathways. In response to ERS, the pERK pathway rapidly attenuates protein translation, the IRE1 pathway upregulates expression of factors involved in ER-associated degradation in order to degrade unfolded proteins, while the ATF6 pathway increases expression of ER chaperones such as GRP78/BiP and calreticulin in order to refold unfolded proteins accumulated in the ER. These pathways are designed to relieve the accumulation of misfolded ER proteins; however, when these pathways are overwhelmed by sustained ERS, then initiates proapoptotic pathways $(25,27-30)$. GRP78, which is localized in the ER, is an important molecular chaperone, and it has been used extensively as an indicator of the induction of ERS (31). p-ERK is known as an initial, crucial protector for survival during even mild stress (9). p-ERK is transmembrane protein in the ERS, which plays a signal transduction role. In the inactive state, p-ERK and two other ERS sensors (IRE-1 and ATF6) are associated with the ER chaperone GRP78/BiP. When unfolded and/or misfolded proteins increase, they dissociate from GRP78/BiP and activate downstream molecules (14). 

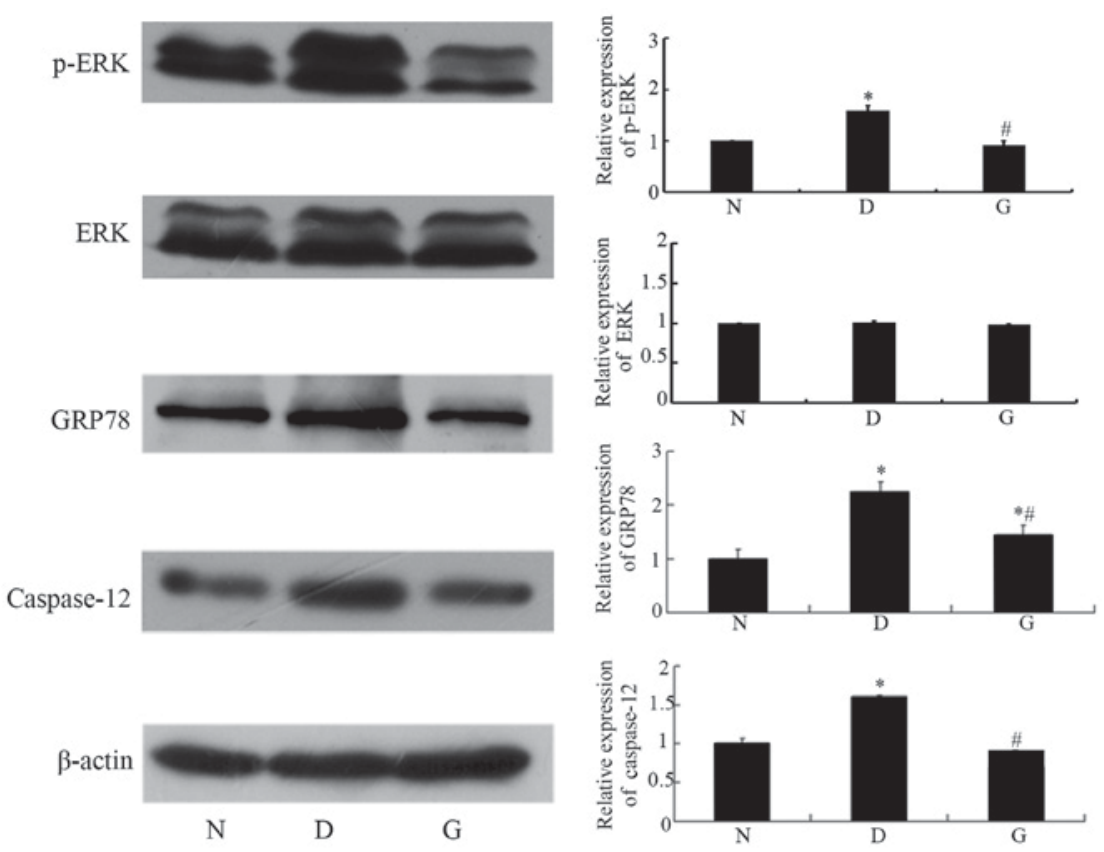

Figure 3. GSPE inhibits the expression of GRP78, p-ERK and Caspase-12. Western blotting analysis for GRP78, p-ERK, and Caspase-12, and quantification of corresponding protein levels. Animals were divided into three group: The control group (N), the DM group (D), the GSPE group (G). Data in the expression of GRP78, p-ERK, ERK and Caspase-12 are expressed as mean \pm SD levels relative to $\beta$-actin. The histogram means the relative protein expression of each group to the $\mathrm{N}$ group, $\mathrm{N}$ group was set to 1 . In the relative expression of ERK, D group was $1.01 \pm 0.02, \mathrm{G}$ group was $0.98 \pm 0.01$. No significant difference was observed between the two groups $(\mathrm{P}>0.05)$. In the relative expression of $\mathrm{p}$-ERK, $\mathrm{D}$ group was $1.59 \pm 0.09, \mathrm{G}$ group was $0.92 \pm 0.08$. This indicates that $\mathrm{p}$-ERK levels changed without any effect on the level of normal ERK. In the relative expression of GRP78, D group was $2.22 \pm 0.21, \mathrm{G}$ group was $1.44 \pm 0.19$. In the relative expression of Caspase-12, D group was $1.60 \pm 0.03, \mathrm{G}$ group was $0.70 \pm 0.01$. Compared with the $\mathrm{N}$ group, the expressions of GRP78, p-ERK and Caspase-12 in the D group were significantly increased. Compared with the D group, the expression of GRP78, p-ERK and Caspase-12 were significantly decreased in the G group. ${ }^{*} \mathrm{P}<0.05$ compared with the $\mathrm{N}$ group; ${ }^{*} \mathrm{P}<0.05$ compared with the $\mathrm{D}$ group. $\mathrm{DM}$, diabetes mellitus; GSPE, grape seed proanthocyanidin extracts; $\mathrm{p}$-ERK, phosphorylated-extracellular signal-regulated kinase; GRP78, glucose-regulated protein 78.
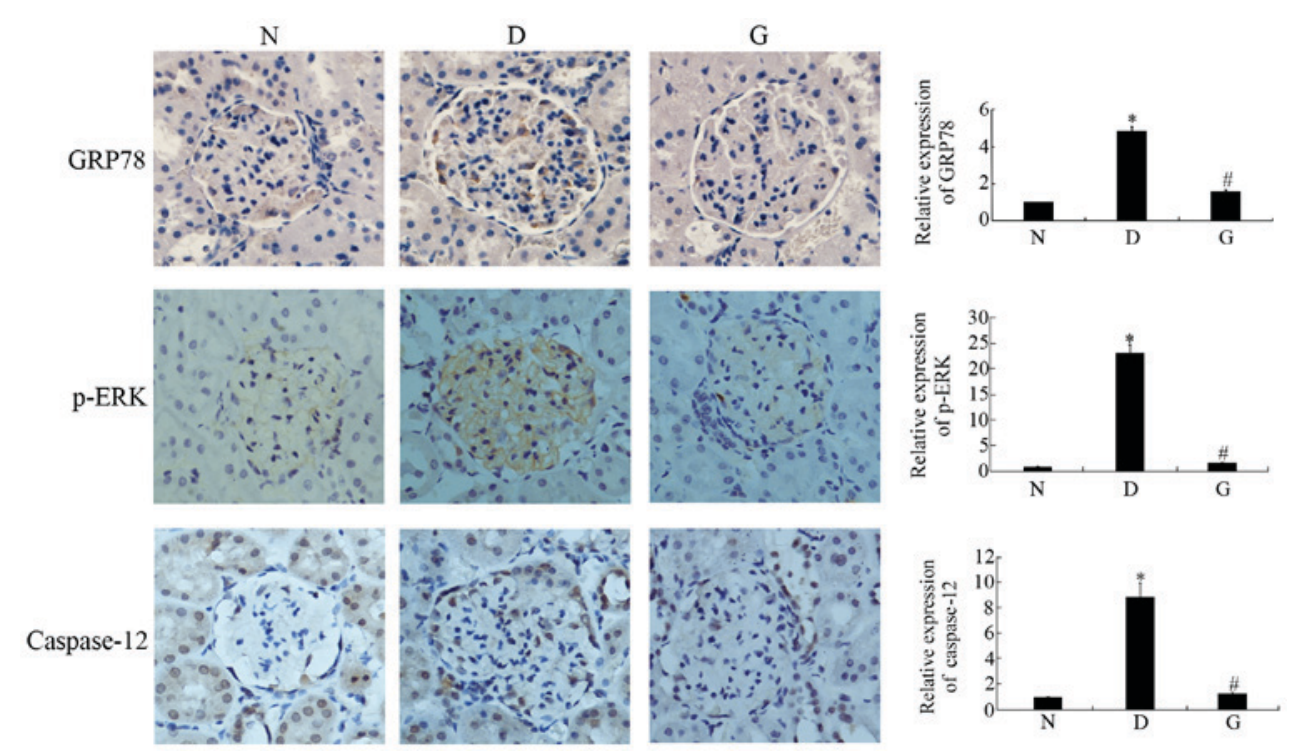

Figure 4. GSPE inhibits the expression of GRP78, p-ERK and Caspase-12. Immunohistochemical staining of GRP78, p-ERK and Caspase-12 of kidney and their measurement of the intensity of corresponding protein in the immunohistochemical staining. Animals were divided into three group: the control group $(\mathrm{N})$, the DM group (D), the GSPE group (G). The grown granules reprehensive the positive cells. Original magnification, x1,000. The expression of GRP78, p-ERK and Caspase-12 were significantly increased in D group compared with N group and decreased in G group compared with D group. Data are presented as means $\pm \mathrm{SD}$. The histogram means the relative protein expression of each group to the $\mathrm{N}$ group, $\mathrm{N}$ group was set to 1. In the relative expression of GRP78, $\mathrm{D}$ group was $4.85 \pm 0.24, \mathrm{G}$ group was $1.57 \pm 0.11$. In the relative expression of $\mathrm{p}$-ERK, $\mathrm{D}$ group is $23.3 \pm 1.60, \mathrm{G}$ group is $1.70 \pm 0.06$. In the relative expression of Caspase-12, D group was $8.90 \pm 1.10$, G group was $1.30 \pm 0.09$. " $\mathrm{P}<0.05$ compared with the $\mathrm{N}$ group; ${ }^{\prime} \mathrm{P}<0.05$ compared with the $\mathrm{D}$ group. DM, diabetes mellitus; GSPE, grape seed proanthocyanidin extracts; p-ERK, phosphorylated-extracellular signal-regulated kinase; GRP78, glucose-regulated protein 78.

Caspase-12 plays a key role in ERS-induced apoptosis (32). Caspase-12 is localized only in the ER, and it is a marker of apoptosis. It has been demonstrated that Caspase-12 mediated apoptosis was specific to the ER, and Caspase-12 
cannot be activated when apoptosis occurs via membrane or mitochondrial targets (33). In brief, GRP78 and p-ERK are markers of ERS, and Caspase-12 is a marker of ERS-induced apoptosis (34).

In our study, the expression of ERS markers, including GRP78, p-ERK and Caspase-12, was upregulated in DN. GRP78 and p-ERK are two different proteins that lead to ERS. The increase of the two proteins in diabetic nephropathic rats showed that ERS had indeed occurred. The results of the TUNEL assay indicated the occurrence of apoptosis in the diabetic nephropathic kidneys. Compared with the normal group, the expression of Caspase-12 in the DM group and the GSPE group was significantly increased, while it was significantly decreased in the GSPE group compared with the DM group. It has been demonstrated that Caspase-12-mediated apoptosis is specific to the ER, and Caspase-12 cannot be activated when apoptosis occurs via membrane or mitochondrial targets (35). Therefore, it was shown that ERS-mediated apoptosis occurred via the Caspase-12 pathway in the DM group. GRP78, p-ERK, and Caspase-12 were significantly lower in the GSPE group, which showed that GSPE has a protective effect against DN, which is achieved by attenuating the ERS-mediated apoptosis via the Caspase-12 pathway.

A large number of evidence suggests that ERS plays an important role in the development and progression of DN, and that reducing ERS may thwart the kidney disease progression (30). In this study, we concluded that GSPE can protect the renal function in DN. The mechanism is related to the attenuation of ERS-induced apoptosis by the Caspase-12 pathway in STZ-induced DN. It may be a novel therapeutic approach in DN. In our study, we only selected a few representative proteins, such as GRP78, p-ERK, and Caspase-12. We hope to be able to investigate other ERS markers such as the splicing of XBP1, the cleavage of ATF6, ATF4 and CHOP induction, the phosphorylation of eIF2 alpha etc in further study to find out whether there are other mechanisms exist in DN, in order to better serve the clinic.

\section{Acknowledgements}

The authors gratefully acknowledge the assistance of Tao Peng in contributing to the revision of the language of the article.

\section{Funding}

This study was supported by Projects of Medical and Health Technology Development Program in Shandong Province (grant no. 2017WS305) and the Science Foundation of Shandong University (grant no. 260110175616015).

\section{Availability of data and materials}

All data generated or analyzed during this study are included in this published article.

\section{Authors' contributions}

ZG, GL, ZH and XL participated to the conception and design of the study, data analysis interpretation and drafting of the manuscript. ZG, WS, BC and PZ contributed to data acquisition and analysis. ZG and XL participated in the drafting of the manuscript and substantive revisions of the important contents of the manuscript. All authors read and approved the final manuscript.

\section{Ethics approval and consent to participate}

The current study was approved by the Animal Ethics Committee of Shandong University, Jinan, Shandong, China (no. DWLL-2013-053).

\section{Consent for publication}

Not applicable.

\section{Competing interests}

The authors declare that they have no competing interests.

\section{References}

1. Najafian B and Mauer M: Progression of diabetic nephropathy in type 1 diabetic patients. Diabetes Res Clin Pract 83: 1-8, 2009.

2. Gilbert RE and Cooper ME: The tubulointerstitium in progressive diabetic kidney disease: More than an aftermath of glomerular injury? Kidney Int 56: 1627-1637, 1999.

3. Verzola D, Gandolfo MT, Ferrario F, Rastaldi MP, Villaggio B, Gianiorio F, Giannoni M, Rimoldi L, Lauria F, Miji M, et al: Apoptosis in the kidneys of patients with type II diabetic nephropathy. Kidney Int 72: 1262-1272, 2007.

4. Liu GH, Sun Y, Li Z, Song T, Wang H, Zhang Y and Ge Z: Apoptosis induced by endoplasmic reticulum stress involved in diabetic kidney disease. Biochem Biophys Res Commun 370: 651-656, 2008.

5. Kumar D, Zimpelmann J, Robertson S and Burns KD: Tubular and interstitial cell apoptosis in the streptozotocin-diabetic rat kidney. Nephron Exp Nephrol 96: e77-e88, 2004.

6. Liang SH, Zhang W, McGrath BC, Zhang P and Cavener DR: PERK (eIF2alpha kinase. is required to activate the stress-activated MAPKs and induce the expression of immediate-early genes upon disruption of ER calcium homoeostasis. Biochem J 393: 201-209, 2006.

7. Pabla N and Dong Z: Cisplatin nephrotoxicity: Mechanisms and renoprotective strategies. Kidney Int 73: 994-1007, 2008.

8. Schroder M and Kaufman RJ: The mammalian unfolded protein response. Annu Rev Biochem 74: 739-789, 2005.

9. Szegezdi E, Logue SE, Gorman AM and Samali A: Mediators of endoplasmic reticulum stress-induced apoptosis. EMBO Rep 7: 880-885, 2006.

10. Cheng M, Gao HQ, Xu L, Li BY, Zhang H and Li XH: Cardioprotective effects of grape seed proanthocyanidins extracts in streptozocin induced diabetic rats. J Cardiovase Pharmacol 50: 503-509, 2007.

11. Li BY, Cheng M, Gao HQ, Ma YB, Xu L, Li XH, Li XL and You BA: Back-regulation of six oxidative stress proteins with grape seed proanthocyanidin extracts in rat diabetic nephropathy. J Cell Biochem 104: 668-679, 2008.

12. Li XH, Xiao YL, Gao HQ, Li B, Xu L, Cheng M, Jiang B and Ma Y: Grape seed proanthocyanidins ameliorate diabetic nephropathy via modulation of levels of AGE, RAGE and CTGF. Nephron Exp Nephrol 111: e31-e41, 2009.

13. Song Y, Li C and Cai L: Fluvastatin prevents nephropathy likely through suppression of connective tissue growth factor-mediated extracellular matrix accumulation. Exp Mol Pathol 76: 66-75, 2004.

14. van der Kallen CJ, van Greevenbroek MM, Stehouwer CD and Schalkwijk CG: Endoplasmic reticulum stress-induced apoptosis in the development of diabetes: Is there a role for adipose tissue and liver? Apoptosis 14: 1424-1434, 2009.

15. Dronavalli S, Duka I and Bakris GL: The pathogenesis of diabetic nephropathy. Nat Clin Pract Endocrinol Metab 4: 444-452, 2008. 
16. Liu Y and Freedman BI: Genetics of progressive renal failure in diabetic kidney Disease. Kidney Int Suppl: S94-S97, 2005.

17. Luo ZF, Feng B, Mu J, Qi W, Zeng W, Guo YH, Pang Q, Ye ZL, Liu L and Yuan FH: Effects of 4-phenylbutyric acid on the process and development of diabetic nephropathy induced in rats by streptozotocin: Regulation of endoplasmic reticulum stress-oxidative activation. Toxicol Appl Pharmacol 246: 49-57, 2010.

18. Negis Y, Aytan N, Ozer N, Ogru E, Libinaki R, Gianello R, Azzi A and Zingg JM: The effect of tocopheryl phosphates on atherosclerosis progression in rabbits fed with a high cholesterol diet. Arch Bioch Biophysics 450: 63-66, 2006.

19. Reed MJ, Meszaros K, Entes LJ, Claypool MD, Pinkett JG, Gadbois TM and Reaven GM: A new rat model of type 2 diabetes: The fat-fed, streptozotocin-treated rat. Metabolism 49: 1390-1394, 2000.

20. Srinivasan K, Viswanad B, Asrat L, Kaul CL and Ramarao P: Combination of high-fat diet-fed and low-dose streptozotocin-treated rat: A model for type 2 diabetes and pharmacological screening. Pharmacol Res 52: 313-320, 2005.

21. Cedó L, Castell-Auví A, Pallarès V, Blay M, Ardévol A, Arola L and Pinent M: Grape seed procyanidin extract modulates proliferation and apoptosis of pancreatic beta-cells. Food Chem 138: 524-530, 2013.

22. Choi AY, Choi JH, Yoon H, Hwang KY, Noh MH, Choe W, Yoon KS, Ha J, Yeo EJ and Kang I: Luteolin induces apoptosis through endoplasmic reticulum stress and mitochondrial dysfunction in Neuro-2a mouse neuroblastoma cells. Eur J Pharmacol 668: 115-126, 2011.

23. Tsutsumi S, Gotoh T, Tomisato W, Mima S, Hoshino T, Hwang HJ, Takenaka H, Tsuchiya T, Mori M and Mizushima T: Endoplasmic reticulum stress response is involved in nonsteroidal anti-inflammatory drug-induced apoptosis. Cell Death Differ 11: 1009-1016, 2004.

24. Song S, Chew C, Dale BM, Traum D, Peacock J, Yamazaki T, Clynes R, Kurosaki T and Greenberg S: A requirement for the p85 PI3K adapter protein BCAP in the protection of macrophages from apoptosis induced by endoplasmic reticulum stress. J Immunol 187: 619-625, 2011.
25. Cybulsky AV: Endoplasmic reticulum stress, the unfoldedprotein response and autophagy in kindey diseases. Nat Rev Nephrol 13: 681-696, 2017.

26. Taniguchi $\mathrm{M}$ and Yoshida H: Endoplasmic reticulum stress in kidney function and disease. Curr Opin Nephrol Hypertens 24: 345-350, 2015.

27. Kim R, Emi M, Tanabe K and Murakami S: Role of the unfolded protein response in cell death. Apoptosis 11: 5-13, 2006.

28. Masanori K: Endoplasmic reticulum stress in the kidney. Clin Exp Nephrol 12: 317-325, 2008.

29. Cunard R and Sharma K: The endoplasmic reticulum stress response and diabetic kidney disease. Am J Physiol Renal Physiol 300: F1054-F1061, 2011.

30. Fan Y, Lee K, Wang N and He JC: The role of endoplasmic reticulum stress in diabetic nephropathy. Curr Diab Rep 17: 17, 2017.

31. Lakshmanan AP, Thandavarayan RA, Palaniyandi SS, Sari FR, Meilei H, Giridharan VV, Soetikno V, Suzuki K, Kodama M and Watanabe K: Modulation of AT-1R/CHOP-JNK-Caspase12 pathway by olmesartan treatment attenuates ER stress-induced renal apoptosis in streptozotocin-induced diabetic Mice. Eur J Pharm Sci 44: 627-634, 2011.

32. Szegezdi E, Fitzgerald U and Samali A: Caspase-12 and ER-stress-mediated apoptosis: The story so far. Ann NY Acad Sci 1010: 186-194, 2003

33. Ohse T, Inagi R, Tanaka T, Ota T, Miyata T, Kojima I, Ingelfinger JR, Ogawa S, Fujita T and Nangaku M: Albumin induces endoplasmic reticulum stress and apoptosis in renal proximal tubular cells. Kidney Int 70: 1447-1455, 2006.

34. Gao Z, Liu G, Hu Z, Li X, Yang XD, Jiang B and Li XH: Grape seed proanthocyanidin extracts protect against cisplatin-induced nephrotoxicity by attenuating endoplasmic reticulum stress-induced apoptosis. Mol Med Rep 9: 801-807, 2014.

35. Nakagawa T, Zhu H, Morishima N, Li E, Xu J, Yankner BA and Yuan J: Caspase-12 mediates endoplasmic-reticulum-specific apoptosis and cytotoxicity by amyloid-beta. Nature 403: 98-103, 2000. 